Revue Interventions économiques

Papers in Political Economy

$55 \mid 2016$

D'un régionalisme à l'autre : intégration ou interconnexion?

\title{
La réunification coréenne : quel est le scénario le plus plausible?
}

Korean Reunification: What Is the Most Plausible Scenario?

Joseph H. Chung et Cheolki Yoon

\section{(2) OpenEdition}

Journals

Édition électronique

URL : http://journals.openedition.org/interventionseconomiques/2896

DOI : 10.4000/interventionseconomiques.2896

ISBN : 1710-7377

ISSN : $1710-7377$

Éditeur

Association d'Économie Politique

Référence électronique

Joseph $\mathrm{H}$. Chung et Cheolki Yoon, « La réunification coréenne : quel est le scénario le plus plausible ?», Revue Interventions économiques [En ligne], 55 | 2016, mis en ligne le 29 juin 2016, consulté le 10 décembre 2020. URL : http://journals.openedition.org/interventionseconomiques/2896 ; DOI : https:// doi.org/10.4000/interventionseconomiques.2896

Ce document a été généré automatiquement le 10 décembre 2020.

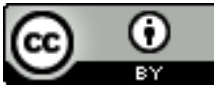

Les contenus de la revue Interventions économiques sont mis à disposition selon les termes de la Licence Creative Commons Attribution 4.0 International. 


\section{La réunification coréenne : quel est le scénario le plus plausible?}

Korean Reunification: What Is the Most Plausible Scenario?

Joseph H. Chung et Cheolki Yoon

1 La réunification coréenne a été un des sujets les plus étudiés et discutés en Corée depuis la division de la péninsule en deux pays en 1945. Il y a, dans de nombreuses universités, des programmes d'études sur la Corée du Nord et la réunification coréenne; il y a des instituts publics et privés de recherche sur ces sujets; il y a même un ministère de la Réunification. En outre, depuis l'année dernière, il y a une Commission préparatoire pour la réunification sous le contrôle direct de la présidente Park Geun-hye. Tout ceci est bien compréhensible, étant donné l'importance de la réunification coréenne tant pour la sécurité et le développement de ces deux sociétés que pour la stabilité et la prospérité de l'Asie du Nord-Est (Chung, J., 2015).

2 Plusieurs scénarios ont été proposés pour la réunification coréenne, mais selon nous, aucun d'eux n'est réalisable à moins d'articuler davantage leurs différentes dimensions politique, économique et militaire et de spécifier les nombreux facteurs qui peuvent influer sur celles-ci. Nous voulons contribuer aux débats sur la réunification coréenne en examinant des scénarios existants. Grosso modo, les scénarios de la réunification coréenne qui, par le passé, ont été discutés et évalués ont favorisé deux directions : le scénario de l'absorption et celui de l'intégration évolutive avec le consentement des deux Corées. Or, le premier se divise en deux sous-scénarios: celui sans consentement des parties et celui qui se réalise avec leur consentement. Le scénario de l'intégration évolutive avec consentement se caractérise par un processus de réunification par étapes. Dans cet ouvrage, nous visons à examiner ces trois scénarios de la réunification coréenne, en particulier sous l'angle de l'économie politique. Ce faisant, nous identifierons les obstacles et les limites intrinsèques à ces scénarios et proposerons ainsi quelques points de repère pratiques qui nous permettront de mieux comprendre la réalité toujours très incertaine d'une réunification de la péninsule coréenne. 


\section{La réunification par l'absorption sans le consentement de la Corée du Nord}

D'abord, il y a deux possibilités de réaliser la réunification coréenne sans consentement de la Corée du Nord : par la guerre ou à la suite de l'effondrement de son régime politique.

\subsection{Une guerre intercoréenne}

Historiquement, la guerre a été l'option choisie par la Corée du Nord en 1950 donnant lieu à la Guerre de Corée qui s'est terminée en 1953, sans réussir la réunification de la péninsule. Cette option n'a cependant pas été rejetée par le président Rhee Syng-man (1948-1960) de la Corée du Sud, mais le pays n'a jamais eu les capacités militaires pour se lancer dans un nouveau conflit ni le soutien des Américains. Malgré quelques escarmouches et quelques affrontements militaires plus sérieux entre les deux pays, la guerre totale ne fut pas répétée. Il parait raisonnable de supposer que la probabilité d'unifier la péninsule par une guerre intercoréenne est aujourd'hui très faible. Il y a plusieurs facteurs qui l'expliquent. En premier lieu, la Corée du Nord est trop puissante sur le plan militaire pour envisager une victoire rapide et facile de la Corée du Sud même avec l'aide des États-Unis ${ }^{1}$; il ne faut pas oublier que la Corée du Nord est bien équipée non seulement en armements traditionnels, mais aussi en armes nucléaires sans compter des missiles à courte et moyenne portée et la possession probable de missiles intercontinentaux (Institut pour l'éducation sur la réunification, 2016). En deuxième lieu, une guerre intercoréenne pourrait évoluer de façon à inviter l'intervention des forces d'autodéfense japonaise, de l'armée chinoise et des forces armées américaines. Il est très possible que les forces alliées sud-coréennes, étatsuniennes et japonaises puissent remporter la victoire, mais une chose est certaine : l'économie sud-coréenne, voire une bonne partie des économies de l'Asie de l'Est, en subirait de lourdes conséquences, avec possiblement des impacts importants sur l'économie globale. Bref, unifier les deux Corées par la guerre est simplement un scénario très difficile à imaginer.

\subsubsection{L'effondrement interne spontané de la Corée du Nord}

Le deuxième scénario est celui qui résulterait de l'effondrement du régime de Kim Jongun. Mais un problème se pose, à savoir, comment évaluer la probabilité d'un tel effondrement, quand nous ne disposons pas vraiment d'informations fiables sur la situation économique et politique interne de la Corée du Nord. Qui plus est, selon certaines recherches (Lankov, 2010 ; Lee, S., 2008), il apparaît de plus en plus clairement que la probabilité d'un effondrement spontané est faible. En définitive, un tel scénario n'est possible qu'en cas de révolte du peuple nord-coréen ou d'un coup d'État militaire. Cela dit, le système de contrôle du peuple paraît toujours fonctionner, notamment en raison de la "politique de songun" qui accorde aux forces militaires une position dominante au sein des institutions politiques de l'État. Ensuite, la concentration systémique du pouvoir autour d'une seule personne, élaborée par Kim Il Sung à partir de ses analyses de la transition de l'autorité au sein des régimes communistes et de la chute du bloc soviétique, semble laisser peu de place à l'émergence de conflits au sein de l'élite politique. Ajoutons que la «sanctification » ou la « déification » de la famille Kim, fait du jeune dirigeant Kim Jong-un, un être à part au sein de l'élite nord-coréenne, quasiment 
intouchable (Steele 2008: 137-142). C'est ainsi que malgré les importantes difficultés économiques, dont la "grande famine» des années 1990 qui aurait fait possiblement entre 600000 et un million de morts, le régime s'est maintenu en place sans qu'il y ait une crise visible à l'intérieur de la classe dirigeante, alors qu'il n'y a plus maintenant d'indicateurs montrant une possible aggravation de la situation économique au cours des dernières années. Enfin, ce scénario place l'effondrement nord-coréen, avant tout, dans le contexte d'un échec du transfert du pouvoir, alors que le jeune leader, Kim Jong-un, semble avoir réussi à consolider son pouvoir depuis son arrivée en 2012, à la surprise d'ailleurs de plusieurs observateurs, même si on peut encore douter de sa longévité potentielle à la tête du pays (Cha, 2016).

6 À l'extérieur qui aurait intérêt à voir le régime s'effondrer ? Pas la Chine en tout cas ! Pour deux principales raisons. D'abord, la Corée du Nord joue le rôle de zone «tampon » empêchant l'extension de la zone d'influence américaine jusqu'à la frontière sinocoréenne. Ensuite, l'effondrement abrupt du pays fondé par Kim Il-sung entraînerait un grand nombre de réfugiés, possiblement vers la Chine, lui imposant une lourde charge économique (Fondacaro, 1997; Roy, 2004 ; Yoo, 2005 ; Zhang, 2007). Le Japon ? Selon Onozuka (2006:12-14), si Tokyo est en faveur d'une réunification qui mènerait à la mise en place d'un régime aligné sur Washington, ce serait beaucoup moins le cas si ce régime s'alignait sur Beijing compte tenu des rivalités régionales. Quant aux États-Unis, ils font preuve, comme la Corée du Sud d'ailleurs, d'une attitude ambivalente. D'une part, ils craignent la réunification de la péninsule coréenne dans de telles conditions en raison du fardeau financier qu'ils devraient supporter, en partie du moins, avec la Corée du Sud compte tenu du niveau de développement actuel de la Corée du Nord et d'autre part, il y aurait toujours la possibilité que de la réunification émerge un régime inamical aux intérêts américains (Odgaard, 2007 ; Pillsbury, 2007).

\subsection{L'effondrement de la Corée du Nord provoqué par des forces externes}

7 Il y a toujours la possibilité que l'effondrement de la Corée du Nord soit initié par des forces externes hostiles au régime. En Corée du Sud, malgré les efforts des 15 dernières années en matière de coopération avec le Nord, que ce soit dans un cadre gouvernemental ou dans le cadre d'initiatives privées, on constate la présence de certaines forces sociopolitiques qui agissent de façon à provoquer l'effondrement du régime de Kim Jong-un. À cet égard, les signes sont nombreux et soulignent l'influence politique de ces forces comme en témoignent, au niveau gouvernemental, la coupure récurrente des canaux de coopération depuis la présidence de Lee Myung-bak (2008-2013), la fermeture de la station touristique du Mont Kumgang ou bien encore la fermeture de la zone industrielle de Kaesong en février 2016 par la présidente Park Geunhye ; le recourt de plus en plus rapide aux sanctions financières et économiques à l'égard du régime nord-coréen lorsque celui-ci ne respecte les résolutions du Conseil de Sécurité ; et, enfin, le développement de plans militaires à caractère plus offensifs, voire préemptifs comme le projet d'opérations militaires « Kill Chain » qui vise à détruire les missiles nordcoréens avant leur lancement et celui de la "décapitation» du régime en visant des installations militaires précises, voire l'élimination physique des hauts dirigeants avec des forces spéciales (The National Post, 2016). Enfin, des initiatives privées, comme l'envoi de ballons de propagande vers la Corée du Nord par des opposants au régime 
nord-coréen (malgré la désapprobation toute relative de Séoul), se multiplient, mais les États-Unis semblent avoir mis fin au financement de ces groupes principalement actifs en Corée du Sud (Halvorssen et Llyod, 2014).

De telles actions sont-elles en mesure de provoquer l'effondrement spontané du régime nord-coréen? On peut en douter fortement. Depuis que la Corée du Nord en mesure de produire des armes nucléaires, le climat de peur que l'on pouvait noté à Pyongyang et associé, entre autres, aux exercices militaires conjoints Corée du Sud - États-Unis s'est, nous semble-t-il, largement dissipés. Les sanctions financières et économiques n'ont pas empêché de nouveaux investissements chinois, voire russes, de limiter la portée de cesdites sanctions sur le régime qui a fait du développement de l'économie une priorité nationale (sans compter la vigueur d'une économie de marché souterraine). En un mot, les mesures prises pour favoriser, voire accélérer l'effondrement du régime du Nord, ne fonctionnent pas.

\section{La réunification par l'absorption avec consentement de la Corée du Nord}

9 Nous avons vu que le scénario de la réunification coréenne par l'absorption sans le consentement de la Corée du Nord est peu probable. Mais alors, est-il possible d'envisager un scénario de réunification d'absorption, mais avec consentement, comme cela s'est en quelque sorte produit lors de la réunification des deux Allemagnes? Ou pour dire les choses autrement, le scénario allemand peut-il s'appliquer la péninsule coréenne? Les explications offertes dans plusieurs études (Coghlan, 2008 ; Hunt, 2006 ; Kelly, 2011 ; Kim, A., 2013 ; Wolf, 1998 ; Kim, Y-Y., 2010 ; Kim, Y-M., 2008) nous amènent à penser que non.

10 En premier lieu, la réunification allemande a été précédée de l'«Ostpolitik», soit la politique de l'Est, de Willy Brandt des années 1970. Cette politique de normalisation des relations entre l'Allemagne de l'Ouest et les pays communistes était fondée, entre autres, sur les mesures suivantes: 1) la reconnaissance réciproque de la souveraineté des deux Allemagnes; 2) l'échange mutuel des ambassades dès 1972 en vertu du Traité fondamental et ; 3) la coopération économique qui signifiait des aides généreuses de la part de l'Allemagne de l'Ouest d'une valeur de près de 2 milliards de dollars par an durant la période de 1972 à 1989 (Wolf, 1998).

11 En Corée, il n'y a pas eu une politique semblable, ni une situation géostratégique favorisant la normalisation des relations bilatérales sauf pour un court moment durant lequel le président Kim Dae-jung (1998-2003), s'inspirant justement de l'Ostpolitik, a mis en place sa " politique de la main tendue », mieux connue sous son appellation anglaise, " Sunshine Policy ", la politique du "rayon de soleil », une " Nordpolitik » ayant comme cible la Corée du Nord. Les relations entre les deux Corées ont toujours été caractérisées par le soupçon, la méfiance, avec une bonne dose d'hostilité : la Guerre de 1950-1953 a été suivie d'un armistice qui n'a jamais mené à un traité de paix, les deux pays étant encore aujourd'hui officiellement en guerre. De plus, l'aide économique offerte par Séoul s'est limitée à 400 millions de dollars sur huit ans (Chung, S., 2015), et en retour, Pyongyang n'a jamais témoigné de réelles intentions de rapprochement, refusant constamment de respecter ses engagements.

12 En deuxième lieu, la réunification allemande fut essentiellement le résultat d'une décision commune. La politique de l'Est de Brandt, l'aide économique soutenue, l'expansion des 
canaux de communication entre les deux pays et, enfin, les liens historiques unissant le peuple allemand ont favorisé, avec la fin de la guerre froide, le processus de réunification. Dans ces conditions, la dissolution des institutions politiques de l'Allemagne de l'Est et la reconnaissance de la légitimité de celles de l'Allemagne de l'Ouest devenaient naturelles. Pour les Allemands de l'Ouest, s'unir avec les Allemands de l'Est était nécessaire pour que le peuple allemand uni puisse détenir une influence à la mesure de son poids économique et politique dans le processus d'intégration et la création de l'Union européenne.

La situation sur la péninsule coréenne est à des années-lumière du cas Allemand, notamment en raison de l'étanchéité de la frontière et du refus des deux gouvernements de tout rapprochement, même au niveau des citoyens. En Corée du Sud, par exemple, selon la Loi sur la sécurité nationale, il est illégal d'aller en Corée du Nord sans autorisation du gouvernement ou de faire des commentaires positifs sur ce pays. Il existe également des lois semblables en Corée du Nord. Dans une telle situation, les bons sentiments entre les deux peuples font place depuis la fin de la Guerre de Corée à des jugements forts négatifs de part et d'autre et, en conséquence, cela a mené à l'émergence de deux sociétés distinctes ayant, avec les années, toujours moins de choses en commun. Les deux gouvernements allemands ont au contraire encouragé, leurs citoyens à se rapprocher et à se connaître. N'oublions pas que la réunification allemande fut adoptée le 18 mars 1990 en grande partie en vertu d'une élection générale qui s'est tenue simultanément sur les deux territoires. Ce serait, en partie du moins, le fruit des efforts de rapprochement entamés dans les années 1970.

En troisième lieu, la réunification allemande a été possible grâce à la vision à long terme et à la qualité des dirigeants, en particulier Willy Brandt (chancelier de l'Allemagne de l'Ouest, 1969 à 1974) et Helmut J. M. Kohl (chancelier de l'Allemagne de l'Ouest, 1982 à 1990 et de l'Allemagne unie, 1990 à 1998). En témoigne la série de traités qui ont pavé la voie de la réunification : le Traité de réunification du 31 août 1990, le Traité d'intégration monétaire et économique du 8 mai 1990 et la Déclaration sur la réunification du 3 octobre 1990. Les deux Corées auraient besoin, dans ce scénario, de dirigeants d'une telle stature, mais compte tenu du climat d'hostilité, il est peu probable qu'il en soit ainsi.

En quatrième lieu, la réunification allemande était d'autant plus facile que les pays européens étaient en faveur de la réunification, alors que le mouvement Perestroika de Gorbatchev en 1985 et celui de Glasnost en 1986 ont grandement facilité la réunification allemande à laquelle ne s'est pas opposée l'URSS. Dans le cas de la réunification coréenne, un tel consensus régional n'existe pas.

On peut tirer les leçons suivantes de la réunification allemande. D'abord, il faut qu'il y ait une idéologie commune : les Allemands de l'Est voulaient la démocratie tout comme les Allemands de l'Ouest. Soumis depuis des décennies à une propagande anticapitaliste, antijaponaise et antiaméricaine, il n'est pas certain que les Coréens du Nord veuillent vivre sous un régime dominé par les symboles capitalistes honnis de Pyongyang comme les chaebol (des immenses conglomérats industriels et financiers qui dominent la vie économique et qui n'ont pas nécessairement bonne presse même en Corée du Sud) et une élite politique longtemps condamnée par Pyongyang comme étant pro-japonaise et corrompue. La deuxième leçon est que la réunification doit être désirée également par les deux peuples séparés. En principe, $67 \%$ des Coréens du Sud sont en faveur de la réunification (sans qu'un scénario ne soit spécifié), mais $56 \%$ pensent que la réunification apportera à leur pays plus de pertes que de gains (Lee, 2006). De plus, la proportion de Sud-Coréens qui appuient la réunification diminue continuellement, en particulier chez 
les jeunes (Park et al., 2014). Enfin, la troisième leçon est que les deux peuples une fois unifiés puissent partager une vision commune de leur histoire et de leur avenir à l'image des Allemands et de leur rôle dans une Europe dédiée à la paix et à la prospérité. Il n'y a pas sur la péninsule coréenne une vision commune d'une Corée réunifiée et pourtant, celle-ci pourrait contribuer activement à la sécurité et la prospérité de la région à titre de puissance économique et militaire moyenne.

Il y a une dernière raison qui rend presque impossible d'envisager pour la péninsule coréenne le scénario de réunification «à l'allemande». N'oublions pas que la réunification allemande s'est déroulée pacifiquement, mais malgré cela, d'après une étude (Wolf, 1998), elle aurait coûté 2000 milliards de marks, sans compter l'aide économique de plusieurs milliards offerte par l'Allemagne de l'Ouest à l'Est durant les années 1970 et 1980. Quel serait le coût de la réunification coréenne dans l'hypothèse où la Corée du Nord consentirait à se faire absorber par le Sud? Il n'est pas difficile de supposer qu'il serait certainement beaucoup plus élevé, compte tenu du degré élevé de pauvreté en Corée du Nord.

La population de la Corée du Nord, en ce moment-ci, est la moitié de celle de la Corée du Sud (25 millions contre 50 millions), alors qu'en 1989, la population de l'Allemagne de l'Est n'était que $26 \%$ de celle de l'Allemagne de l'Ouest (16 millions contre 60 millions). En outre, le PIB par habitant de la Corée du Nord en 2013 représentait à peine $10 \%$ de celui de la Corée du Sud (2 000 dollars contre 20000 dollars américains), alors qu'en 1989, le revenu par habitant de l'Allemagne de l'Est représentait $72 \%$ de celui de l'Allemagne de l'Ouest (18 000 dollars contre 25000 dollars). En un mot, les Sud-Coréens devront soutenir beaucoup plus de Nord-Coréens, de surcroît beaucoup plus pauvres que les Allemands de l'Est. (Kelly, 2011).

19 Ce n'est pas tout, il faudra moderniser les infrastructures, intégrer le système nordcoréen de production et de distribution des biens et des services au système sud-coréen et créer littéralement un réseau d'institutions financières sur le territoire de l'ancienne Corée du Nord. Il faudra établir une série de politiques monétaires et fiscales en mesure d'éliminer les relents et restants d'un régime stalinien et de son idéologie du Juche (ayant très peu évolué depuis les années 1960) pour faire place à un régime démocratique, libéral et capitaliste. Dans cette optique, il faudra mettre sur pied une politique du travail en vue d'assurer l'intégration des travailleurs Nord-Coréens à l'économie de marché, ceux-ci n'ayant à peu près aucune connaissance des règles de fonctionnement d'une telle économie. Ce n'est pas seulement la Corée du Nord qui sera transformée du tout au tout, mais également la société sud-coréenne qui devra absorber les coûts phénoménaux des investissements publics et privés nécessaires à la reconstruction du pays (Hunt, 2006; Kelly, 2011; Koh, 2012; Wolf, 1998).

On ne peut ignorer non plus le problème des armes nucléaires et les armes de destruction massive, notamment les stocks importants d'armes chimiques que détient la Corée du Nord aujourd'hui. Leur élimination sera dispendieuse et leur sécurisation tout autant pour éviter que des stocks tombent entre les mains de groupes terroristes ou entre les mains de factions rebelles de l'Armée nord-coréenne opposées à la réunification. D’autre part, l'instabilité sociale ou militaire pourrait exiger des forces de stabilisation dont le coût serait probablement refilé à la Corée du Sud.

21 Donc, quel serait, donc, au final le coût de la réunification coréenne? Il est difficile d'arriver à un chiffre précis et les évaluations varient considérablement d'une étude à l'autre, notamment en raison du degré d'incertitude lié au déroulement du processus de 
réunification, mais il est généralement reconnu que ces coûts seraient beaucoup plus élevés que pour la réunification allemande, notamment pour les raisons déjà citées. Selon des études récentes (Lee, Y., 2012; Shin, 2011), la réunification de la péninsule coréenne coûterait entre 1000 à 5000 milliards de dollars, soit approximativement entre $80 \%$ et 390 \% du PIB de la Corée du Sud en 2014. Selon une autre étude de 2009, il en coûterait 1500 milliards pour amener le PIB per capita de la Corée du Nord à $60 \%$ de celui de la Corée du Sud sur une période de 10 ans. Mais selon une autre étude, il en coûterait entre 2000 et 5000 milliards pour amener le niveau de vie moyen des Nord-Coréens à $80 \%$ du niveau de vie des Sud-Coréens. Il se pourrait que cette dernière estimation soit celle qui se rapproche le plus de la réalité (Lankov, 2013 : 235-237), mais face à une telle situation, il ne fait aucun doute que l'aide des pays étrangers sera nécessaire pour réussir ce que l'Allemagne a fait au cours des 25 dernières années.

\section{Réunification d'intégration évolutive}

La réunification coréenne par le truchement d'un processus d'absorption, qu'elle soit avec ou sans le consentement de la Corée du Nord, nous apparaît peu probable, mais dans tous les cas, la réunification entraînera un grand fardeau pour la société sud-coréenne. Il faut donc explorer d'autres scénarios. Notons que la plupart des présidents de la Corée du Sud ont proposé des idées sur la réunification de la péninsule. Rhee Syng-man (1948-1960) voulait unifier la péninsule par force. Le premier ministre Chang Myon (1960-1961) a, pour sa part, suggéré à l'ONU une élection générale sous la supervision de cette dernière. Le général Park Chung-hee, au cours de son règne dictatorial (1962-1979) a suggéré en 1972 une réunification des deux régimes autoritaires basée sur l'autonomie, la paix et la solidarité. Ces propositions demeurent largement inarticulées. Il faut attendre la proposition du président Roh Tae-woo (1988-1993) pour avoir un scénario plus articulé. Roh annonçait, lors d'un discours à l'Assemblée nationale le 11 septembre 1989, ce qu'on a appellé la Formule de réunification de la communauté nationale (National Community Unification Formula)qui préconisait une forme de confédération Nord-Sud comme étape préliminaire à la réunification et basée sur la paix, la dénucléarisation, la non-agression et la coopération. Roh faisait donc allusion à une forme de coexistence coopérative entre deux régimes à l'idéologie antagoniste (Choi, 2001).

Cette formule comporte trois étapes d'évolution des relations intercoréennes vers la réunification : l'étape de la réconciliation et de la coopération, celle du Commonwealth coréen (Nambuk yonhap) et, enfin, celle de la réunification. Ces étapes devraient être franchies en respectant une série de directives dont l'une d'elles précise que le processus de réunification doit être guidé par les principes de la démocratie, de l'indépendance et de la paix. Le scénario de Roh fut repris et développé par le président Kim Dae-jung (1988-2003), mais cette fois en mettant de l'avant les principes suivants : la non-agression, la non-intervention des pays étrangers dans le processus de réunification, la coopération mutuelle et surtout la reconnaissance mutuelle de la souveraineté des régimes. Pour Kim, une première étape dans la coopération économique mutuelle devait prendre la forme de sa «Sunshine Policy» alors que par la suite, les deux parties, le Nord et le Sud, tout en respectant leur souveraineté, devraient préparer des institutions nécessaires à la réunification. Il y aurait donc une longue série des rencontres à tous les niveaux dont des rencontres au sommet et des rencontres ministérielles, et la mise en place d'un Conseil 
intercoréen, le tout avec l'objectif d'établir une forme de confédération précédant une dernière étape, celle de la création d'un gouvernement national.

Le scénario proposé par la Corée du Nord est similaire à la formule du président Roh (ce qui n'a pas empêché Pyongyang de la rejeter catégoriquement en affirmant que seule sa propre «formule» tenait la route). Elle date de 1974 lorsque Kim Il-sung formula sa vision de la réunification de la péninsule qui prendrait la forme de la "République démocratique confédérale de Koryo » (en l'honneur de la dynastie du même nom qui a unifié la péninsule en 936). Le sixième congrès du Parti des travailleurs en faisait la politique officielle de la Corée du Nord en octobre 1980 et celle-ci se base sur des principes similaires à ceux annoncés dans la Formule du président Roh, soit la nonintervention des pays étrangers, la paix, le respect mutuel de souveraineté et la coopération. Elle ne spécifie pas qu'il y ait un processus de réunification par étapes, mais en revanche elle propose une série d'institutions précises à établir (Park, 2014). Ce scénario préconise le principe d'un gouvernement central qui s'occupe de la défense nationale et de la diplomatie et de deux gouvernements régionaux (au Sud et au Nord) qui prennent en charge le «bien-être du peuple ». Il s'agit d'un régime pour un seul peuple et un seul pays, mais formé de deux gouvernements régionaux et de deux régimes distincts, une démocratie capitaliste au Sud et un régime socialiste populaire au Nord, avec un Conseil suprême national confédéral formé de représentants des deux Corées en plus de représentants de la diaspora coréenne.

Avec la mise en valeur de sa Nordpolitik et de sa Sunshine Policy, Kim Dae-jung aurait pu enfin réaliser les projets de réunification de Roh Tae-woo et de Kim Il-sung en signant, lors du sommet intercoréen historique entre lui et Kim Jong-il de juin 2000, la Déclaration commune 6.15 qui était en quelque sorte une synthèse de la Formule de Roh et de la Confédération Koryo de Kim. Cette déclaration se basait sur des principes chers à Kim Dae-jung, à Roh et Kim Il-sung : la non-intervention des pays étrangers, la paix et le respect des régimes politiques des deux parties. Grâce à la Sunshine Policy mise en application après la Déclaration commune de 6.15 , la coopération économique intercoréenne a pris des formes concrètes, notamment, la construction de la zone industrielle de Kaesong et le développement touristique du Mont Kumgang.

Le président Rho Moo-hyun (2003-2008) a succédé à Kim Dae-jung, mais sa politique de rapprochement, bien qu'elle respecte celle mise en place par son prédécesseur, n'a pas fonctionné aussi bien, et ce en raison, d'une part, des tensions entre la Corée du Nord et les États-Unis entourant les armes nucléaires et le retrait en 2003 de la Corée du Nord du Traité de non-prolifération nucléaire signant ainsi l'arrêt de mort de l'Accord de Genève de 1994 et, d'autre part, des difficultés de mise en œuvre de l'accord, notamment le processus de la vérification de l'abandon du programme nucléaire nord-coréen et la construction des réacteurs à eau légère par les États-Unis. Les pourparlers à six (la Chine, la Corée du Nord, les États-Unis, la Corée du Sud, la Russie et le Japon) ont suivi de 2003 à 2007, mais l'absence de résultats concrets et le refus de la Corée du Nord de respecter ses engagements ont amené les négociations dans une impasse des plus totales (Park et Chung, 2013). Le gouvernement sud-coréen continua cependant le dialogue avec le Nord durant cette période, ce qui a mené au deuxième sommet intercoréen entre Roh Moohyun et Kim Jong-il où ils signèrent une déclaration sur la paix qui devait amener la rédaction d'un traité de paix pour remplacer l'armistice de 1953. Le rapprochement des deux Corées a pris un virage abrupt en 2008 avec la mesure prise le 24 mai (mesure dite 5.24) par le président Lee Myung-bak (2008-2013) après qu'une touriste sud-coréenne ait 
été abattue par un soldat nord-coréen sur le site touristique du Mont Kumgang. Cette mesure a empêché toutes relations intercoréennes et depuis, les tensions militaires se sont accrues sur la péninsule avec le second essai nucléaire de 2009, le naufrage de la corvette Cheonan en mars 2010 et le bombardement de l'île Yeonpyeong en novembre 2010 par l'artillerie nord-coréenne. Le gel des relations Nord-Sud a continué sous le régime de la présidente Park Geun-hye, et ce, malgré des gestes de bonne volonté de sa part, comme sa déclaration de Dresden du 28 mars 2014 et l'instauration de la Commission préparatoire à la réunification. Park faisait ainsi preuve de sa détermination de ne pas répondre aux provocations de Pyongyang en tendant la main, tout en menant parallèlement des actions visant à affaiblir le Nord, notamment la fermeture de la zone industrielle de Kaesong en février 2016 à la suite des tirs de missiles et de l'essai nucléaire de janvier par le Nord.

Les expériences coréennes en matière de réunification permettent d'entamer les observations suivantes. En premier lieu, la réunification par l'absorption avec ou sans le consentement de la Corée du Nord apparaît très difficile, sinon impossible. En deuxième lieu, il faut donc chercher un scénario fondé sur une non-absorption avec un consentement mutuel pour une forme de réunification. Le scénario de la Formule du président Roh et celui de la Confédération Koryo de Kim Il-Sung sont précisément des scénarios de non-absorption reposant sur un consentement mutuel. En troisième lieu, une synthèse de ces deux scénarios qui formait le cœur de la Nordpolitk de Kim Dae-jung et de Roh Moo-hyun n'a pas duré longtemps à cause de la persistance de la méfiance et du soupçon mutuels, voire par moment de la défiance entre les deux pays.

Il faut donc chercher un scénario qui n'envisage pas une réunification à court terme, mais qui l'envisage comme un processus d'intégration progressive des activités politiques et économiques des deux Corées et qui, si possible, et si nécessaire, se terminerait par une réunification véritable. Ce scénario, fondé sur l'intégration évolutive, est le plus réaliste compte tenu du fait qu'il est le seul qui ait été admis historiquement par les deux Corées malgré des différences de détails. En outre, en dépit des obstacles vécus et prévus, ce scénario apparaît relativement plus réalisable que les autres scénarios envisagés précédemment et il correspond davantage aux deux besoins cruciaux de la Corée du Nord : la garantie du régime et la survie économique (Kim, K., 2012).

\section{Pour un scénario viable de la réunification par une intégration évolutive}

Eu égard aux expériences historiques et aux difficultés examinées, nous suggérons les principes suivants afin d'envisager un scénario viable pour la réunification coréenne. D'abord, il faudrait distinguer la notion d'intégration et celle de réunification. Par la première, on entend un processus par lequel les deux Corées coopèrent sur les plans politique et économique selon des normes et des règles communes, alors que pour la seconde, on entend la fusion des deux régimes en un seul, qui pourrait prendre la forme d'un régime fédératif doté d'un gouvernement fédéral et d'États régionaux ou un État unitaire doté d'un seul gouvernement central. En deuxième lieu, la réunification devrait se faire dans une perspective fonctionnaliste et liée à une série d'étapes qui débuterait par une intégration des activités économiques et ensuite politiques des deux parties, pour ensuite faire avancer progressivement la réunification. En troisième lieu, le processus doit prendre le temps nécessaire pour permettre à l'économie nord-coréenne 
de se développer et de rattraper davantage l'économie sud-coréenne et, par conséquent, diminuer considérablement les coûts de la réunification. premier temps, il est essentiel qu'un traité de paix soit signé entre la Corée du Nord et les États-Unis et il faut cesser les sanctions financières et économiques contre le régime de Kim Jong-un à condition bien sûr que ce dernier se débarrasse de ses armes nucléaires ou du moins arrête le développement et la production d'armes nucléaires additionnelles. Pour y arriver, il est souhaitable que les États-Unis entreprennent un dialogue bilatéral avec Pyongyang en vue de normaliser leur relation, car aussi longtemps que l'état de guerre existe et que les sanctions persistent, il est difficile d'assurer un développement normal de l'économie nord-coréenne et de garantir la sécurité de la région.

Dans un deuxième temps, il faudrait que les pays de la région, dans une perspective à long terme, acceptent la réunification de la Corée. Le Japon pourrait hésiter à le faire, considérant sa rivalité potentielle avec une Corée réunifiée. Cependant, d'un autre côté, celle-ci donnerait aux firmes japonaises un accès plus facile à un marché élargi sans compter les facilités d'accès accrues aux marchés chinois et russe, des facteurs positifs pour le Japon. Dans le cas de la Chine, elle devrait être en faveur de la réunification coréenne à long terme, car cette dernière lui permettrait de protéger et d'accroître ses investissements, de diminuer les tensions militaires et de se libérer du souci d'accommoder les réfugiés nord-coréens qui pourrait déferler en Chine dans le cas d'un processus de réunification qui ferait suite à l'effondrement du régime nord-coréen. D'un autre côté, la Chine pourrait bien s'inquiéter de la présence américaine à sa frontière avec la Corée, il serait alors important que les forces armées américaines quittent la Corée ou du moins se relocalisent au sud de la zone démilitarisée, voire au sud de la péninsule pour ne pas hausser l'insécurité de Beijing.

d'envergure. Ils n'accepteront pas une Corée réunifiée dotée de l'arme nucléaire ; ils accepteront difficilement la présence dans une Corée réunifiée d'un régime non démocratique et ils s'opposeront à une alliance militaire sino-coréenne après la réunification. L'attitude des Américains à l'égard de la réunification pourrait se refroidir si les sentiments antiaméricains s'intensifient (Haselden, 2002; Kim, 2005), mais, aussi longtemps que la Corée réunifiée demeure une alliée fiable, Washington ne s'y opposera pas. La Corée pourrait même jouer un rôle de médiateur intermittent entre les États-Unis et la Chine. En ce qui concerne la Russie, il apparaît assez clairement qu'elle devrait accepter la réunification de la péninsule, car cela signifie pour Moscou un accès plus facile au marché pétrolier coréen et japonais, grâce au gazoduc reliant les gisements de Sibérie à l'économie de la Corée et éventuellement à celle du Japon.

Nous appuyons ainsi un scénario qui va procéder par étapes et par intégration fonctionnelle. Le processus de la réunification devrait procéder via la coopération commerciale et économique, la création d'une union douanière, l'intégration financière et économique, la création d'un marché commun et, enfin, la réunification. Nous croyons que la coopération commerciale et économique entre le Sud et le Nord pourrait se développer grâce à plusieurs facteurs. D'abord, il y a une forte complémentarité entre ces deux régions. Le Sud peut fournir le capital et les technologies, le Nord peut offrir une main-d'œuvre abondante, bien éduquée, motivée et à bon marché. Le Nord détient des matières premières évaluées à plus de 6000 milliards de dollars (Chung, S., 2015) et la Corée du Nord pourra également faciliter l'accès des biens et des services sud-coréens aux 
marchés chinois, russe et même européen via " la nouvelle route de soie " reliant l'Asie à l'Europe. Il ne faut pas oublier que les entreprises sud-coréennes ont déjà une bonne expérience en Corée du Nord dans le cadre de la zone industrielle de Kaesong et du site touristique du Mont Kumgang. Faut-il noter également que les Coréens du Sud et du Nord parlent la même langue et partagent une culture et des traditions similaires, facilitant sans doute la communication nécessaire entre gens d'affaires. Il serait alors très important que la Corée du Nord puisse mettre en place un environnement règlementaire et politique favorable aux investissements étrangers. Il faudrait peut-être un régime hybride caractérisé par une coexistence du marché libre et du secteur public afin d'assurer un système de distribution équitable, compatible au socialisme, ce qui a déjà été constaté, dans une forme limitée, avec l'émergence et l'élargissement d'un marché privé (notamment les Jang-madang, des places de marché comme les marchés agricoles publics ou les marchés clandestins qui sont au cœur de l'économie de marché souterraine) et les zones économiques spéciales (Cha, 2007 ; Institut pour l'éducation sur l'unification, 2016; Kim, Choo et Im, 2010).

34 La coopération économique intercoréenne pourrait prendre plusieurs formes. La Corée du Sud pourrait investir dans les infrastructures probablement en collaboration avec la Chine, le Japon, la Russie, les États-Unis et bien d'autres pays, en particulier pour développer des aéroports, des chemins de fer, des routes et des ports. Il faudrait également investir dans la modernisation des systèmes de production et de distribution des biens et des services. Ajoutons que la Corée du Nord a déjà établi une douzaine de zones économiques spéciales à travers son territoire ayant pour but d'assurer un développement régional équilibré. Les entreprises sud-coréennes pourraient profiter de ces ZES, notamment si des mesures incitatives pour les investisseurs étrangers sont offertes.

La création d'une union douanière comporte deux mesures : un accord de libre-échange intercoréen et des droits de douane communs sur les biens importés par les deux Corées. Il faut se rappeler que des droits de douane communs pourraient simplifier la gestion du commerce international des deux Corées. La troisième étape, notamment celle de l'intégration financière et économique doit commencer par la détermination d'un taux de change et éventuellement l'adoption d'une même monnaie et d'une coordination serrée de la politique monétaire. L'intégration économique devrait prendre la forme de la coordination du système de production et de celui de distribution.

36 La quatrième étape, celle de la formation du marché commun consiste à assurer la mobilité libre des travailleurs, et ceci nécessite l'intégration des marchés du travail des deux Corées. La cinquième et la dernière étape, celle de l'intégration politique, est la plus difficile à réaliser. Par intégration politique on entend le processus d'établir des institutions ayant pour but d'effectuer les fonctions gouvernementales, à savoir, la législation, la gestion du système judiciaire et l'exécution administrative. Bref, l'intégration politique est la création d'un mécanisme de gouvernance, d'un gouvernement. Il peut y avoir différents modèles de gouvernance : le régime fédératif, le régime confédératif ou le régime unitaire. La Corée du Nord favorise un modèle fédéral doté d'un gouvernement fédéral et de deux gouvernements régionaux. Quant à la Corée du Sud, il n'y a pas de préférence publicisée. Cependant, il est bien possible qu'à long terme, le régime unitaire démocratique soit l'objectif ultime de la réunification.

De toute manière, il est trop tôt pour avoir une idée claire sur le modèle de gouvernement d'une Corée réunifiée. Il faut entreprendre des études conjointes intercoréennes avant 
d'envisager une gouvernance plausible. Cependant, une chose certaine est que le choix du régime politique devrait passer par un rapprochement des idéologies des deux Corées qui apparaissent pour le moment incompatibles (Chamberlain, 2004). D'abord, la possibilité de choisir l'idéologie du Juche par la société sud-coréenne est nulle. Ensuite, l'adoption nord-coréenne du régime de la démocratie libérale du Sud signifie que l'économie nordcoréenne doit passer une période de la transformation du régime socialiste au régime capitaliste (Campos et Coricelli, 2002). L'imposition d'une seule idéologie dès le début est réellement impossible et ne correspond pas non plus à l'esprit démocratique. Le rapprochement idéologique devrait être inévitablement le résultat d'un long processus de contacts et d'échanges accrus entre les deux sociétés (Goodby, 2006; Jagar, 2006).

\section{Conclusion}

En conclusion, la réunification de deux Corées est certainement souhaitable non seulement pour la sécurité et le développement de ces deux sociétés, mais également pour la stabilité et la prospérité de la région de l'Asie du Nord-Est. Les choix de la réunification de la péninsule coréenne se résument en deux scénarios de base : celui par un processus d'absorption avec consentement ou sans de la Corée du Nord et celui d'intégration évolutive. Le scénario d'absorption sans consentement de la Corée du Nord n'est possible qu'en cas d'une guerre intercoréenne ou d'effondrement du régime du Nord, ce qui semble improbable dans un avenir proche. La réunification par un processus d'absorption avec le consentement du Nord n'est pas réalisable pour le moment, à cause des idéologies politiques séparant les deux régimes et la peur de la part de la Corée du Nord de se faire assimiler à l'idéologie capitaliste du Sud, sans compter le coût d'une telle réunification.

Il faut donc trouver un scénario qui permet une coexistence des deux régimes comme une étape temporaire et en même temps qui permet le chemin vers la paix et la prospérité des deux Corées. Nous croyons que le scénario d'intégration évolutive serait le plus réaliste. Ce scénario a les caractéristiques suivantes. En premier lieu, la réunification se fait par des étapes d'intégration fonctionnelle: la coopération commerciale et économique, la création d'une union douanière, l'intégration financière et économique, la formation d'un marché commun et la création d'une gouvernance politique. Jusqu'à l'étape de la création d'un marché commun, les deux Corées collaborent en tant que deux pays souverains et conservent leur idéologie et leur régime politique. C'est l'étape de la création de la gouvernance politique qui va décider la forme du gouvernement soit le fédéralisme, soit le confédéralisme ou soit le gouvernement unitaire. D'après nous, il est souhaitable de former un gouvernement unitaire. Cependant, ceci nécessite un rapprochement idéologique entre deux parties. Ce qui n'est pas facile est de prévoir le temps requis pour la réalisation de ce scénario d'intégration évolutive ; une chose certaine, il faudra un long temps, probablement, une couple de décennies. Le scénario d'intégration évolutive en tant que tel nous semble ainsi relativement faisable. De plus, dans des cas imprévus, comme l'effondrement du Nord, la planification et l'implémentation étape par étape de ce scénario pourraient contribuer à réduire des charges, soit économiques ou sociales, qui suivent. 


\section{BIBLIOGRAPHIE}

Campos, Nauro, F. et Coricolli, Fabrizio (2002), Growth in Transition: What We Know, What We Don't and What We Should, William Davidson Working Paper, $\mathrm{n}^{\circ}$ 470, 75p.

Cha, Moon Seok (2007), " "Markets" and "Market Economy” in North Korea: Money Substitues Suryung ", Discourse 201, vol. 10, n², pp. 77-121.

Cha, Victor D. (2016), « The North Korea Question », Asian Survey, vol. n² 2, vol. 56, mars-avril, pp. 243-269.

Chamberlain, Paul F. (2004), « Cultural Dimensions of Korean Reunification: Building a Unified Society ", International Journal on World Peace, vol. 21, n 1, pp. 3-42.

Chamberlain, Paul F. (2007), « Today's Korea Questions: Establishing a Peace on a Denuclearized Korean Peninsula », in Bruce E. Bechtol (dir.), The Quest for a United Korea: Strategies for the Culture and Inter-Agency Process, Quantico, V. A., Marine Corps University Foundation, pp. 25-65.

Choi, Jong-gu (2001), « Socio-economic Approach for Softening Border: Implication of Experience in the Korean Peninsula », East Asia Review, vol. 8, n 1, pp. 19-30.

Chung, Joseph H. (2015), « Unification de deux Corées: Pourquoi? Quand? Comment? », Texte présenté lors d'une Conférence MKLCC-OAE, CEIM, UQAM, 9 octobre.

Chung, Seung-im (2015), « Il n'y aura pas de progression sans rencontre au sommet ... : Entrevue avec l'ancien ministre de la réunification Chung, Seh-hyun », The Korea Times, 13 août. Récupéré en ligne le 18 janvier 2015: http://www.hankookilbo.com/v/59903a4ab25e4a2db9e8fa7dd53d1507

Coghlan, David (2008), Prospect from Korean Reunification, Carlisle Barracks, PA, U.S. Army War College, avril. Récupéré en ligne le 18 janvier 2015 : http:// www.strategicstudiesinstitute.army.mil/pdffiles/PUB859.pdf

Fondacaro, Steve A. (1997), « An Alternative Scenario for the Reunification of Korea », USAWC Strategy Research Project, Carlisle Barracks, U.S. Army War College, 10 mai, 43 pages.

Goodby, James E. (2006), Creating a Peace Regime in Korea, Brookings Institute, 30 mai. Récupéré en ligne le 18 janvier 2015: http://www.brookings.edu/research/opinions/2006/05/30northkoreagoodby

Halvorssen, Thor et Alexander Llyod (2014), « We Hacked North Korea With Ballons and USB Drives », The Atlantic, 15 janvier. En ligne : http://www.theatlantic.com/international/ archive/2014/01/we-hacked-north-korea-with-balloons-and-usb-drives/283106/

Haselden, Carl E. (2002), « The Effects of Korean Unification on the U.S. Military Presence in Northeast Asia », Parameters, vol. 32, n 4, pp. 120-132.

Hunt, Jennifer (2006), The Economics of German Reunification, McGill University and NBER, février. Récupéré en ligne le 18 janvier 2015 : http://www.rci.rutgers.edu/ jah357/Hunt/ Transition_files/german_unification.pdf

Hwang, Kwan (1999), « Neutralization: An All-Weather Paradigm for Korea Reunification », Asian Affairs, vol. 25, n 4, pp.195-207. 
Institut pour l'éducation sur la réunification (2016), Comprendre la Corée du Nord, Séoul, Ministère de la Réunification, République de Corée, 364p.. Récupéré le 7 juin 2016: http://

nkinfo.unikorea.go.kr/nkp/pblictn/viewPblictn.do?

pageIndex=1\&pblictnId=64\&origin $\mathrm{d}=0 \mathrm{C} 0001 \&$ tabType=pblictn_pic

Jagar, Sheila Miyoshi (2007), « Time to End the Korean War: The Korean Nuclear Crisis in the Era of Unification », Japan Focus, vol. 4, n 10. Récupéré en ligne le 18 janvier 2015: http:// www.japanfocus.org/-Sheila\%20Miyoshi-Jager/2255/article.html

Kelly, Robert E. (2011), « The German-Korea Unification Parallel », Korea Journal of Defence Analysis, vol. 23, nं 4, pp. 457-472.

Kim, A Joo (2013), Analysis of the Reunification Experiences of Germany, Vietnam and Yemen: Finding Ideal Process and Conditions toward Successful Reunification of Korea, Summer Honors Research, Sweet Briar, VA, Sweet Briar College. Récupéré en ligne le 18 janvier 2015:

http://www.sbc.edu/sites/default/files/Honors/AJooKim.July31_0.pdf

Kim, Christine (2013), « Korean Unification May Cost South Korea 7 percent of GDP: Ministry », Reuters, $1^{\mathrm{er}}$ janvier. Récupéré en ligne le 18 janvier 2015 : http://www.reuters.com/article/uskorea-north-unification-idUSBRE90004F20130101

Kim, Jiyul (2001), « Continuity and Transformation in Northeast Asia and the End of American Exceptionalism: A Long Range Outlook and US Policy Implications ", Korean Journal of Defence Analysis, vol. 13, n 1, pp. 229-261.

Kim, Jiyul (2005), « Pan-Korean Nationalism, Anti-Great power-ism and U.S.-South Korea Relations ", Japan Focus, vol. 3, n 12. Récupéré en ligne le 18 janvier 2015: http:// www.japanfocus.org/-jiyul-kim/1679/article.html

Kim, Kae-dong (2012), Ouverture pour manger, diplomatie nucléaire pour vivre : la diplomatie de la Corée du Nord, Séoul, Salim, 44 pages.

Kim, Yong-Min (2008), «Considération pour l'unification coréenne, compte tenu de l'exemple de l'Allemagne », Langue et littérature allemandes, vol. 22, n 1, pp. 323-342.

Kim, Young-Yun (2010), « Scénario de l'intégration économique des Corées vu à la lumière de la réunification allemande », Économie unifiée, hiver, pp. 76-88.

Kim, Young-Yun, Choo Won-Seo et Im Eul-Chool (2010), Étude sur la coopération intercoréenne pour la circulation des marchandises à la ville de Lajin, Séoul, Institut coréen pour l'unification nationale, $173 p$.

Koh, Il-dong (2012), « Benefits and Cost of Korea's Unification: Major Issues and Possible Responses », 22 octobre. Texte présenté lors d'une conférence IGE/KEXIM/KAS/KDI/PIIE. Récupéré en ligne le 18 janvier 2015: https://www.kdi.re.kr/data/download/attach/9716_2-2.pdf

Lankov, Andrei (2013), The Real North Korea: Life and Politics in the Failed Stalinist Utopia, Oxford, Oxford University Press.

Lankov, Andrei (2010). « Unification: It Will be Hard, It Will be Good, and It Will Happen », The Kookmin Review, n² 216, 3 mai. Récupéré en ligne le 18 janvier 2015 : http:// english.kookmin.ac.kr/site/news/press/8?pn=6

Lee, Cheoleon (2006), « Gallup World Poll: Implications of Reunification of Two Koreas », Gallup, 12 octobre. Récupéré en ligne le 18 janvier 2015 http://www.gallup.com/poll/24949/gallup-worldpoll-implications-reunification-two-koreas.aspx 
Lee, Sangkeun (2008), «A Critical Review of the Predictions of North Korea's Collapse in the 1990s and the 2000s », Unification Studies, vol. 12, n 2, pp. 93-133.

Lee, Younghoon (2012), « The Changing Unification Environment and a New Approach to the Studies of Unification Cost and Policy », North Korean Studies, vol. 16, n 1, pp. 267-291.

Ministère de la Défense nationale, République de Corée (2014), 2014 Defense White Paper, Séoul, 328 pages. Récupéré en ligne le 7 juin 2016: http://www.mnd.go.kr/cop/pblictn/

selectPublicationUser.do?

siteId=mnd\&componentId=14\&categoryId=15\&publicationSeq=672\&pageIndex=1\&id=mnd_050601000000

National Post, The (2016), « North Korea accuses U.S. military and South Korea of planning a 'beheading operation' in annual military exercise », 7 mars. En ligne: http:// news.nationalpost.com/news/world/north-korea-accuses-u-s-miliary-and-south-korea-ofplanning-a-beheading-operation-in-annual-military-exercise

Odgaard, Liselotte (2007), « The Balance of Power in Asia-Pacific Security: US-China Policies on Regional Order », Korean Journal of Defence Analysis, vol. 19, n 1,pp. 29-46.

Onozuka, Takayuki (2006), « Security of Japan and Korean Unification », USAWC Strategy Research Project, Carlisle Barracks, US Army War College. Récupéré en ligne le 18 janvier 2015 : http://www.strategicstudiesinstitute.army.mil/pdffiles/ksil446.pdf

Park, Keun-young et Chung Wook-sik (2013), L'arme nucléaire et la suite, Séoul, Poolbit, 309 pages.

Park, Myung-kyu, et al. (2014), 2014 Survey on Korean Unification, Seoul, Institute for Peace and Unification Studies, Seoul, Seoul National University.

Park, Young Ho (2014), « South and North Korea's Views on the Unification of Korean Peninsula and Inter-Korean Relations ».Texte présenté lors d'une conférence KRIS-Brookings, 21 janvier. Récupéré en ligne le 18 janvier 2015 : http://www.brookings.edu/ /media/events/2014/1/21korean-peninsula-unification/park-young-ho-paper.pdf

Pillsbury, Michael (2007), How to Hedge against Rising China, The ICAS Lectures, Blue Bell, Institute for Corean-American Studies. Récupéré en ligne le 18 janvier 2015: http:// www.icasinc.org/2007/2007s/2007smxp.html

Roy, Danny (2004), « China and the Korean Peninsula: Beijing's Pyongyang Problem and Seoul Hope », Asia Pacific Security Studies, vol. 3, n 1. Récupéré en ligne le 18 janvier 2015: http:// apcss.org/Publications/APSSS/ChinaandtheKoreanPeninsula.pdf

Ruehl, Steven A. (2001), When Korea Reunifies...Will Asians Say Hello or Goodbye to G.I.Joe?Carlisle Barracks, U.S. Army War College, 26 pages.

Shin, Dong-jin (2011), Revue de la littérature en matière du coût de l'unification, Analyses des affaires économiques $n^{\circ}$ 64, Séoul, Bureau du budget de l'Assemblée nationale, 38p.

Steele, Jesse D. (2008), « Negotiating with Deity : Strategies and Influences Related to Recent North Korea Negotiating Behavior ", Pepperdine Dispute Resolution Law Journal, vol. 9, $\mathrm{n}^{\circ}$ 1, pp. 199-146.

Taylor, William J. (2000), « Prospects for Reunification: Producing One Korea is unlike any Other Geopolitical Situation in the Word ", The World and I, vol.15, $\mathrm{n}^{\circ} 6$.

Wolf, Holger (1998), « Korean Unification: Lessons from Germany », in Marcus Noland (dir.), Economic Integration of the Korean Peninsula, Washington D.C., Institute for International Economics, pp. 165-189. 
Yoo, Chan Yul (2005), « Anti-American, Pro-Chinese Sentiment in South Korea », East Asia: An International Quarterly, vol. 22, n 1, pp. 18-32.

Zhang, Quanyi (2007). « What Korean Unification Means to China », NAPSNet Policy Forum Nautilus Institute, 12 octobre. Récupéré en ligne le 18 janvier 2016: http://nautilus.org/napsnet/ napsnet-policy-forum/what-korean-unification-means-to-china/

\section{NOTES}

1. Selon le Livre blanc sur la défense de 2014 de la Corée du Sud (Ministère de la Défense nationale, 2014, p. 239), la Corée du Nord possède des troupes évaluées à 1020000 soldats, 7700000 soldats en incluant les troupes de réserve, tandis que la Corée du Sud a 630000 soldats et 3100000 soldats dans ses troupes de réserve. Malgré la difficulté de la Corée du Nord d'opérer ses armements en raison de leur vieillissement et de l'offre instable d'énergie, étant donné la taille de ses troupes et le terrain montagnard, les batailles terrestres prendront du temps à se concrétiser.

\section{RÉSUMÉS}

Dès la division en 1945, la réunification est un des enjeux essentiels entre les deux Corées. Elle est cruciale non seulement pour la sécurité et le développement des deux sociétés, mais également pour la stabilité et la prospérité de l'Asie du Nord-Est. Pendant plus d'un demi-siècle, plusieurs scénarios de réunification ont été envisagés, mais chaque scénario contient ses propres limites et pendant ce temps les tensions persistent toujours sur la péninsule coréenne. Dans cet article, nous nous proposons d'analyser les discours et les perspectives concernant la réunification coréenne et d'identifier un scénario réalisable. Les scénarios existants peuvent être divisés en trois catégories: celui de l'absorption sans consentement mutuel, celui de l'absorption avec consentement mutuel et celui d'une intégration évolutive. À travers cet examen, nous voulons préciser les limites des scénarios existants et proposer quelques points de repère pour concevoir une piste appropriée pour la réunification. Selon nous, le seul scénario réaliste est celui d'une intégration évolutive.

Since the division of Korea in 1945, the Korean reunification has been one of the most strategic issues in two Koreas. It is crucial not only for the security and the evolution of these two societies, but also for the stability and the prosperity of the North-East Asia as a whole. For more than half a century, several reunification scenarios have been presented, but each scenario contains its own limitations and shortcomings, and the tension still persists on the Korean Peninsula. In this article, our aim is to examine various arguments and viewpoints on the Korean reunification and find a more feasible scenario. There are three basic scenarios: the absorption scenario without consensus between the two parties, the absorption scenario with a consensus between two parties, and the evolutional integration scenario. Through this examination, we will evaluate the limitations and shortcomings of existing scenarios and propose the most feasible scenario. We think that the most feasible is the evolutional integration scenario of reunification. 
INDEX

Mots-clés : Corée du Nord, Corée du Sud, intégration évolutive, réunification coréenne

Keywords : evolutional integration, Korean reunification, North Korea, South Korea 